\title{
AUFSÄTZE
}

\section{Paradoxien des Parlamentarismus. Historische und aktuelle Gründe für Fehlverständnisse in Wissenschaft und Öffentlichkeit*}

\author{
Gerhard Loewenberg
}

Parlamenten wird nach wie vor mit Missverständnissen und Misstrauen begegnet. In Deutschland wurde der Reichstag als „Quasselbude“ bezeichnet; es wurde ihm vorgeworfen, nur Parteiengezänk anstatt vernünftiger Lösungen zu produzieren. Aber tief sitzende Skepsis und herabsetzende Bemerkungen über das Parlament gibt es nicht nur in Deutschland, sondern überall: In den Vereinigten Staaten etwa, dem Land mit dem mächtigsten Parlament der Welt, wird der Kongress immer am negativsten unter allen politischen Institutionen bewertet.

Historiker und Politologen haben immer wieder die spezifisch deutschen Faktoren untersucht, die die negativen Beurteilungen über das Parlament in diesem Land erklären könnten. Gewiss muss man sich immer wieder mit der Katastrophe befassen, für die der Reichstag in Flammen ein unvergessliches Symbol ist. Sie war aber bestimmt nicht das unvermeidliche Ergebnis besonderer deutscher Missverständnisse über das Parlament, denn Deutschland konnte auf langjährige Erfahrungen mit Abgeordnetenversammlungen zurückblicken. Diese Erfahrungen gab es sowohl auf der lokalen als auch auf der nationalen Ebene, sowohl in vordemokratischen als auch in demokratischen Zeiten, sowohl in ruhigen als auch in strapaziösen Phasen auf dem Weg zum Nationalstaat, während der Industrialisierung, im Krieg und im Frieden. Deutschland kann eine eindrucksvolle parlamentarische Tradition vorweisen. Wegen vielfacher historischer Brüche sorgt man sich hier aber mehr als in anderen Ländern über öffentliche Missverständnisse gegenüber Parlament und Parlamentarismus.

Die folgenden Überlegungen über das öffentliche Unverständnis und die Geringschätzung des Parlamentes sind nicht auf Deutschland beschränkt. Das Parlament ist eine politische Institution, die immer und überall von ihrem Wesen her gefährdet ist. Deshalb sollen hier einige grundsätzliche Ursachen für das öffentliche Misstrauen gegenüber dem Parlament untersucht werden ${ }^{1}$ - Gründe, die nicht spezifisch für Deutschland sind, aber spezifisch in Bezug auf bestimmte Eigenschaften des Parlaments als Institution.

* Vortrag (in überarbeiteter Form), gehalten auf der Abendveranstaltung der Deutschen Vereinigung für Parlamentsfragen in Berlin am 6. Juni 2007. Eine englischsprachige Version ist in der Zeitschrift Daedalus der American Academy of Arts and Sciences im Sommer 2007 (H. 3) erschienen (S. 55 - 66). Für die Hilfe bei der Übersetzung möchte ich mich bei Sylvia Ulrich bedanken.

1 Vgl. für die Bundesrepublik auch Werner J. Patzelt, Ein latenter Verfassungskonflikt? Die Deutschen und ihr parlamentarisches Regierungssystem, in: PVS, 39. Jg. (1998), S. 725 - 757. 
Parlamente sind angeblich die demokratischste politische Institution. Sie repräsentieren die Bevölkerung, aber die Art und Weise wie sie arbeiten, ist den meisten Menschen ein Rätsel. Weil sie alle Bürger repräsentieren sollen, bestehen Parlamente aus einer großen Anzahl von Mitgliedern. Jedes Mitglied hat ein gleichwertiges Mandat. Insofern ist nicht ohne weiteres zu erwarten, dass 400 oder 500 oder 600 Abgeordnete miteinander Entscheidungen treffen, da eine große Anzahl von stolzen, gleichwertigen Repräsentanten zunächst keinen Grund hat, aufeinander zu hören.

Fakt ist, dass Parlamente verwirrend sind. Sie scheinen gegensätzliche Eigenschaften zu haben, weisen Widersprüche auf. Diese haben ihren Ursprung in der Struktur der Institution selbst. Sie verdeutlichen, warum Parlamente so flächendeckend falsch verstanden und so oft ins Lächerliche gezogen werden. Nachfolgend werden vier Paradoxien identifiziert, die sich aus der Geschichte der Institution ergeben. Sie entstehen aus dem Gegensatz zwischen den Merkmalen ihres Ursprungs im mittelalterlichen Europa, und jenen, die die Institution entwickelt hat, als sie sich dem wandelnden politischen Umfeld anpasste. Die ursprünglichen Merkmale betrachte ich als die genetischen Charakteristika. Sie stehen in vielerlei Hinsicht im Widerspruch zu den später entwickelten.

Das moderne Parlament hat seine Vorgänger in den feudalen Versammlungen des mittelalterlichen Europas. ${ }^{2}$ Auf diese Ursprünge gehen drei Eigenschaften zurück: Erstens war das Parlament eine Versammlung von einflussreichen Feudalherren, die der König von Zeit zu Zeit zusammenrief, um ihr Einverständnis zu finanzieller Unterstützung und militärischer Rekrutierung einzuholen; zweitens waren die Mitglieder dieser Versammlungen Repräsentanten, zum Beispiel von sozialen Klassen oder von lokalen Interessen, und das bestimmte, wer als Mitglied in Frage kam und wie sie ausgewählt wurden; und drittens berieten und verhandelten die Mitglieder mit dem Monarchen. Sie gewährten dem König seine Wünsche und erhielten dafür gesetzliche Erleichterungen. Daraus wird auch die etymologische Wurzel verständlich: Es ging ums Reden (parlare), nicht ums (Mit-)Regieren.

Als diese Versammlungen sich häufiger und regelmäßiger trafen, entwickelten sie Verfahren, um gemeinsame Entscheidungen zu ermöglichen. So wurden diese gelegentlichen Versammlungen der Feudalherren in Parlamente umgewandelt, aber diese waren nicht - noch lange nicht - demokratische Parlamente. Von diesen Ursprüngen her haben Parlamente also folgende genetische Merkmale: (1) Sie sind Versammlungen von einflussreichen Personen; (2) ihre Mitglieder repräsentieren andere; und (3) ihre Mitglieder verhandeln im Interesse ihrer Territorien oder ihrer Stände mit der Regierung. Diese genetischen Eigenschaften ließen Parlamente zu politischen Institutionen werden, die in all ihren weiteren Erscheinungen erkennbar sind.

Anfangs machten die Parlamente ihren Einfluss nur gelegentlich geltend. Mit der Zeit erhoben sie aber Anspruch auf die gesetzgebende Gewalt. Der Begriff „legislature“ tauchte zum ersten Mal Ende des 17. Jahrhunderts in England auf, abgeleitet von dem Wort „legislator“, welches „Gesetzgeber“ bedeutet. Im Oxford English Dictionary wird zum Begriff „legislature“ ausgeführt, dass sich die erste Quellenangabe auf Matthew Hales 1713 veröffentlichte „History of Common Law“ bezieht. Darin findet man den Satz: „Without the

2 Zur Geschichte des Parlamentarismus vgl. auch mit weiteren Nachweisen Werner J. Patzelt, Grundriss einer Morphologie der Parlamente, in: ders. (Hrsg.), Evolutorischer Institutionalismus. Theorie und exemplarische Studien zu Evolution, Institutionalität und Geschichtlichkeit, Würzburg 2007, S. $483-564$. 
concurrent Consent of all Three Parts of the legislature, no (...) law (...) can be made.“ Bemerkenswert ist die Formulierung ,all Three Parts of the legislature“. Das ist ein Hinweis auf das Oberhaus, das Unterhaus und den König, die zusammen den Gesetzgeber bildeten. Dass der Begriff „legislature“ nur noch mit dem Parlament in Verbindung gebracht wurde, war das Ergebnis von Montesquieus knapp vier Jahrzehnte später vorgelegter Gewaltenteilungslehre, mit der er den Machtmissbrauch im Absolutismus verhindern wollte.

Montesquieus Lehre kam in Amerika besonders gut an. In „The Federalist“ Nr. 47 über die Gewaltenteilung zitiert James Madison wiederholt „the celebrated Montesquieu“. Das Wort „legislature“ wurde fortan zur gängigen Bezeichnung für die Kolonialversammlungen in Amerika. Indem sich diese gesetzgebende Kompetenz aneigneten, beschränkten sie die britische Kontrolle. Später wurde „legislature“ auch auf die repräsentativen Versammlungen in Lateinamerika bezogen; diesem Sprachgebrauch wurde in anderen Ländern aber nicht gefolgt. So verwendete man in Deutschland seit dem Mittelalter den Namen Reichstag, mit dem die repräsentative Versammlung bezeichnet wurde. In Russland wurde das Parlament Duma genannt, in Israel Knesset, in Frankreich Nationalversammlung usw. Es ist die Struktur der Institution als Versammlung, die ihren Namen bestimmt. In den meisten Ländern kennzeichnet der Name nicht die Funktion.

Das Verhältnis der Legislative zur Exekutive gestaltete sich in Europa anders als in Amerika. In der Alten Welt entwickelte sich das mittelalterliche Parlament nicht durch die Gewaltenteilung, sondern es wurde langsam ein Instrument zur Demokratisierung der Monarchie. Das Parlament erhielt seine Legitimität durch die Ausweitung des Wahlrechts im 19. Jahrhundert im Laufe der Demokratisierung. Dadurch konnten Volksvertretungen die Autorität des Monarchen in Frage stellen, begrenzen und in vielen Ländern ersetzen. Die Geschichte der Parlamentarisierung der Regierung in Deutschland ist ein Beispiel für ähnliche Entwicklungen anderswo in Europa. Im Ergebnis entstand ein parlamentarisches Regierungssystem, in dem die Gewalten zu einem neuen Dualismus zusammenwuchsen. Formal unterscheidet es sich dadurch vom Präsidialsystem, wie es sich auf dem amerikanischen Kontinent entwickelt hat, wo die Gewalten in Form des alten Dualismus getrennt sind.

Egal ob Parlamente Gesetze machende Gremien im Präsidialsystem oder teils (mit-)regierende Gremien in parlamentarischen Systemen wurden: Ursprünglich nur beratende Versammlungen, wurden sie zu Institutionen, die wichtige politische Entscheidungen trafen. Obwohl sie aus den Feudalgesellschaften im mittelalterlichen Europa stammten, entfalteten sie sich zu einer besonders einflussreichen Institution im Amerika des 18. Jahrhunderts und im nächsten Jahrhundert in vielen europäischen Ländern. Und das wahrscheinlich zweitstärkste Parlament der heutigen Welt existiert in Berlin, auf dem Gelände seiner Zerstörung im Jahre 1933, in einem Land, in dem das Parlament zeitweise verheerender Kritik ausgesetzt war. Weiter bemerkenswert ist, dass die meisten heute anzutreffenden Exemplare dieser Institution erst in der zweiten Hälfte des 20. Jahrhunderts entstanden sind; und es ist kaum erstaunlich, dass die Institution, die aus dem Mittelalter stammt, nun im 21. Jahrhundert schwierigen Anpassungen gegenübersteht.

Ihrem Ursprung nach waren Parlamente keine Entscheidungsgremien, sondern Diskussionsforen. Ihre Wandlung zu wichtigen politischen Entscheidungsträgern hat etliche Widersprüche zur Folge. Diese Widersprüche liegen zwischen den genetischen Merkmalen des Parlaments und jenen Eigenschaften, die Parlamente entwickelten, als sich ihre Aufgaben im Staat ausweiteten. Dieser Wandel ist der Ursprung von Paradoxien. 
Man findet Probleme der Entscheidungsfindung in vielen Gruppen, die sich nicht gern der Hierarchie von Befehlen und Gehorsam unterwerfen. Es ist bekannt, wie schwer es ist, Entscheidungen in Vereinen, Kirchen oder Universitätsfakultäten zu treffen - oder sogar in Familien, die versuchen zu entscheiden, wohin die Urlaubsreise gehen soll. Die Konsequenzen solcher Widersprüche in privaten Gruppen sind aber nicht so deutlich wie in der Öffentlichkeit. Die meisten Menschen denken nie darüber nach, warum gemeinsame Entscheidungen so schwierig sein können. Die Probleme erscheinen nur dann so unerklärlich, wenn sie auf der politischen Ebene auftreten, wo Bürger wenig Erfahrung haben.

Es gibt vier Widersprüche, die aus der Art und Weise entstehen, wie eine mittelalterliche Institution sich an den modernen Staat angepasst hat:

(1) Das Paradox der Hierarchie: Parlamente bestehen darauf, dass jedes ihrer Mitglieder über den gleichen Status verfügt, weil jeder ein Repräsentant der Wählerschaft ist. Aber in allen Parlamenten ist es unverzichtbar, Hierarchien zu bilden, um die Arbeit zu organisieren.

(2) Das Paradox des Abstimmungsmodus: Parlamente behaupten, dass sie auf dem Mehrheitsprinzip fußen. Aber sie nehmen Regeln an, die nicht-mehrheitlich sind, um endloses Abstimmen zu vermeiden.

(3) Das Paradox der Transparenz: Parlamente behaupten, dass ihre Sitzungen öffentlich sind. Aber ihre Entscheidungsprozesse sind für die Allgemeinheit undurchsichtig.

(4) Das Paradox internationaler Vergleichbarkeit: Obwohl Parlamente die besonderen sozialen und kulturellen Eigenschaften ihrer Nation reflektieren sollten, ahmen neue Parlamente nach, was alte Parlamente über parlamentarische Einrichtungen gelernt haben.

Diese Widersprüche sind für die meisten Menschen unerklärlich und stehen der Wertschätzung der Institution im Weg. Sie sollen im Folgenden näher untersucht werden.

\section{Das Paradox der Hierarchie}

Die Autorität eines Parlaments gründet auf seinem Anspruch, die Nation zu repräsentieren. Diese Behauptung beruht ihrerseits auf dem repräsentativen Charakter seiner individuellen Mitglieder. Jedes von ihnen ist, in den bekannten Worten des Artikels 38 des Grundgesetzes, ein „Vertreter des ganzen Volkes, an Aufträge und Weisungen nicht gebunden und nur ihrem Gewissen unterworfen“. Alle Abgeordneten sind in dieser Hinsicht gleichwertig und können deshalb rein formal keine hierarchischen Beziehungen untereinander akzeptieren.

Repräsentation von allen Teilen einer Nation führt überall zu einem großen, schwerfälligen Körper. Es besteht kein eindeutiger Zusammenhang zwischen der Bevölkerungsgröße eines Landes und der Größe seines Parlamentes. Gleiches gilt für das Wachstum der Bevölkerungszahl über die Jahre. Vielmehr werden Unterschiede in der Größe des Parlaments durch verschiedene Konzepte von Repräsentation bestimmt, die durch verschiedene Wahlsysteme umgesetzt werden. Am Beispiel: Die ungarische Nationalversammlung, gewählt durch ein kompliziertes dreiteiliges Wahlsystem, hat 386 Mitglieder für eine Bevölkerung von zehn Millionen, also ein Abgeordneter auf 26.000 Bürger. Im Bundestag mit gegenwärtig 613 Mitgliedern für 82 Millionen Menschen kommt ein Parlamentarier auf 134.000 Personen. In den Vereinigten Staaten repräsentieren 435 Abgeordnete 300 Millionen Menschen, also ein Verhältnis von 1:670.000. US-amerikanische Abgeordnete vertreten also 25-mal mehr Personen als ihre ungarischen Kollegen und fünfmal mehr als die Mitglieder 
des Bundestags. Im 16. Jahrhundert im England von Königin Elizabeth I. wuchs die Mitgliederzahl des Unterhauses von 296 auf 462 und damit doppelt so schnell wie die Gesamtbevölkerung. Im Jahre 1700 hatte England darum mehr Parlamentarier auf sechs Millionen Einwohner gerechnet, als US-Amerikaner in ihrem Repräsentantenhaus heute für eine 50-mal größere Bevölkerung haben.

Die Umsetzung eines sich wandelnden Verständnisses von Repräsentation - nicht das Bevölkerungswachstum allein - führt also zu einer großen Mitgliederzahl. Der erste Gedanke ist nicht, wie das resultierende Organ arbeiten kann. Offensichtlich können 400, 500 oder auch 600 Mitglieder überhaupt nicht handeln - ohne eine Steuerung, mindestens eine Vereinbarung über eine Tagesordnung. Hinzu kommt, dass viele Mitglieder der Versuchung erliegen, der Arbeit auszuweichen, sich um Entscheidungen herumzudrücken, solange es keine Abmachungen zur Verteilung der Aufgaben gibt. Tagesordnung und Arbeitsteilung bedürfen der Organisation und bedeuten letztlich Einschränkungen für das, was jedes Mitglied tun kann. Daraus ergeben sich Ungleichheiten unter den Abgeordneten.

Die Bestimmung der Tagesordnung sowie die Art und Weise, wie Streitpunkte behandelt und Alternativen strukturiert werden, beeinflussen das Endergebnis stark. Weigern sich Abgeordnete, die Aufstellung der Tagesordnung an einen Vorsitzenden oder einen Ausschuss zu delegieren, verläuft die parlamentarische Arbeit unkoordiniert oder gar chaotisch. Das Gleiche gilt für die Organisation der Gesetzgebungsarbeit. Wenn kein Versuch unternommen wird, Verantwortung zu delegieren, sind Abgeordnete versucht, sich vor jeglicher Verantwortung zu drücken.

Am Beispiel: Die Mitglieder der kolonialen Versammlungen in Amerika kannten diese Gegebenheiten. Von 39 Unterzeichnern der Verfassung der Vereinigten Staaten hatten 18 in kolonialen Parlamenten gedient. Aber als Mitglieder des Kontinentalkongresses ignorierten sie ihre früheren Erfahrungen. In der unsteten politischen Situation des revolutionären Amerikas erhielt jeder Abgeordnete eifersüchtig die Vorrechte seines Herkunftsstaates aufrecht. Die nötigen Hierarchieformen akzeptierten sie nicht. Sie waren nicht gewillt, einem von ihnen das Recht, die Tagesordnung zu bestimmen, anzuvertrauen. Folglich litt der Kongress unter einer Unorganisiertheit, die typisch ist für Institutionen, die nur nach dem Mehrheitsprinzip entscheiden. Ein Mitglied beschrieb folgendes Ereignis: Ein Bericht vom "Committee of the Whole" wurde angenommen und im Journal eingetragen, dann vom nächsten Bericht überholt; der zweite wurde daraufhin einstimmig angenommen und eine halbe Stunde später wieder gestrichen. „Wenn ich hinzufüge“, schrieb er, „dass Irritationen dieser Art täglich vorkommen, werden Sie sich nicht wundern, dass ich überall gerne wäre, nur nicht im Kongress. "3

Der Kontinentalkongress weigerte sich im Großen und Ganzen, ständige Ausschüsse einzurichten. Er stützte sich stattdessen auf eine große Anzahl von Sonderausschüssen. Zwischen 1774 und 1788 wurden mehr als 3000 solcher Gremien geschaffen - jedes einzelne mit wenig Autorität und wenig Fachkompetenz. Folglich mussten die meisten Entscheidungen von allen Mitgliedern im Plenum gefällt werden und kamen deshalb nur sehr langsam zustande; der Arbeitsaufwand für jeden Abgeordneten war ungeheuer hoch.

3 Zitiert in Rick K. Wilson, Transitional Governance in the United States: Lessons from the First Continental Congress, in: Gerhard Loewenberg / Peverill Squire / D. Roderick Kiewiet (Hrsg.), Legislatures: Comparative Perspectives on Representative Assemblies, Ann Arbor 2002, S. 299 f. 
Die Entwicklung von Parlamenten in Amerika beweist eine Kontinuität von den kolonialen Versammlungen über die Parlamente der Einzelstaaten nach der Revolution bis hin zum heutigen US-Kongress. Auf dieser Zeitachse war der Kontinentalkongress, so Peverill Squire, eine „evolutionäre Mutation“4. Viele Politologen haben sich mit diesem Mutanten beschäftigt, ähnlich wie Biologen Mutationen studieren, um Erkenntnisse über das „Normale“ zu gewinnen.

Wenn Abgeordnete die Nachteile des Fehlens einer Tagesordnung erleben, suchen sie nach geeigneten Gegenmitteln. Entweder entscheiden sie sich für Steuerungsausschüsse, für Vorsitzende, welche die Beratungen leiten können, oder sie vertrauen bei solchen Entscheidungen den Fraktionsführungen. Wenn Sie gelernt haben, was es heißt, jedem Mitglied eine gleichberechtigte Rolle in jeder Entscheidung einzuräumen, teilen sie die gesetzgebende Arbeit auf. Sie legen die Verantwortung für jedes Gebiet genau fest, auch wenn dies bedeutet, Entscheidungen zu delegieren. Obwohl die Verfahrensordnungen des Preußischen Abgeordnetenhauses und des Reichstags des Kaiserreiches Fraktionen nicht kannten, bezog sich das deutsche Parlament seit den frühen 1850ern darauf, durch Fraktionen sowohl die Tagesordnung auszuhandeln als auch Mitglieder unterschiedlicher Komitees auszuwählen. Fraktionsdisziplin und die Bildung von Ausschüssen sind die gängigen Mittel, um organisatorischen Problemen in Parlamenten zu begegnen. Das bedeutet aber auch, dass der Einfluss individueller Abgeordneter auf Entscheidungen nicht gleich sein wird. Nur sehr unerfahrene parlamentarische Institutionen sträuben sich dagegen.

Fraktionen und Ausschüsse sind die Quelle innerparlamentarischer Hierarchie. Die Forschung hat drei Ursachen für diese Hierarchie identifiziert ${ }^{5}$ :

(1) Abgeordnete delegieren an ihre Fraktionsführungen die Kontrolle über die Tagesordnung, über die Ausschüsse und über die Beziehungen zwischen Kabinett und Parlament. Dadurch gewinnt jede Partei vor den Wählern eine Identifikation, von der die Wiederwahl ihrer Abgeordneten abhängt. Respekt gegenüber Fraktionsführungen ist also Respekt für die Rolle der Parteien in Wahlen.

(2) Die Aufgabenteilung durch Ausschüsse und Arbeitskreise erlaubt es den Abgeordneten, sich zu spezialisieren. Das Parlament kann so das nötige Wissen entwickeln, um in vielen verschiedenen Angelegenheiten gleichzeitig komplexe Entscheidungen zu treffen. Der Respekt gegenüber den Empfehlungen der Ausschüsse und Arbeitskreise ist also Respekt gegenüber Kompetenz und Effizienz.

(3) Die Arbeitsteilung in Ausschüssen und Arbeitskreisen bietet Gelegenheiten zum Verhandeln. Abgeordnete können Entscheidungen, die ihnen wenig bedeuten, an andere Gremien abgeben. Dafür können sie sich in ihren eigenen Ausschüssen mit den Sachfragen befassen, die ihnen besonders wichtig sind. Der Respekt für die Arbeit der Ausschüsse bedeutet die Anerkennung, dass Mitglieder nicht gleich stark an allen Angelegenheiten interessiert sind.

Aus diesen Gründen treten Abgeordnete sich Entscheidungen gegenseitig ab, obwohl sie formal einander gleichgestellt sind. Nur so können sie ihre Arbeit koordinieren und die gewünschten Ergebnisse erzielen. Aber der Konflikt zwischen Statusgleichheit und Hierar-

4 Peverill Squire / Keith E. Hamm, 101 Chambers: Congress, State Legislatures, and the Future of Legislative Studies, Columbus, Ohio 2005, S. 6.

5 Vgl. Suzanne S. Schüttemeyer, Fraktionen im Deutschen Bundestag 1949-1997. Empirische Befunde und theoretische Schlußfolgerungen, Opladen 1998. 
chie verschwindet nie ganz. Es bleibt der Verdacht sowohl bei den Abgeordneten als auch in der Öffentlichkeit, dass sich Vetternwirtschaft und spezielle Interessen durchsetzen. Es scheint, dass Mitglieder, obwohl sie rein nominal gleichgestellt sind, einen sehr unterschiedlich starken Einfluss auf parlamentarische Entscheidungen haben. Fraktions- und Ausschussentscheidungen, die meist nicht öffentlich fallen, und der dezentralisierte Entscheidungsprozess begünstigen die Lobbyisten. Das gilt sowohl für ihren legitimen als auch für ihren unrechtmäßigen Einfluss - wie wir immer wieder beides in den USA und auch in Deutschland erfahren.

Der US-Kongress hat seine dezentralen Strukturen weiter ausgebaut als andere Parlamente. Es gibt 16 Ausschüsse und 87 Unterausschüsse im Senat, 22 Ausschüsse und 135 Unterausschüsse im Repräsentantenhaus. Damit läuft er besonders Gefahr, Partikularinteressen zum Opfer zu fallen - eine Gegebenheit, die in den Vereinigten Staaten heutzutage große Besorgnis erregt. Die Ausschussstruktur des Bundestags ist ebenfalls hochkomplex, wenn man nicht nur seine gegenwärtig 22 ständigen Ausschüsse, sondern auch noch seine fünf Fraktionen und deren Arbeitskreise und Arbeitsgruppen in Betracht zieht.

\section{Das Paradox der Abstimmungen}

Von einem Parlament, das aus gleichberechtigten Mitgliedern in einer demokratischen Gesellschaft besteht, erwartet man Entscheidungen nach dem einfachen Mehrheitsprinzip. Aber Abgeordnete, im Gegensatz zu Bürgern bei allgemeinen Wahlen, treffen laufend Entscheidungen. Dabei wirken sie aufeinander ein, handeln Kompromisse aus - innerhalb der Arbeitskreise, der Fraktionen, der Ausschüsse, selten im ganzen Plenum. Die Schlussabstimmung bestätigt diese Kompromisse, verbirgt aber den zugrunde liegenden Entscheidungsprozess.

Bei jeder Entscheidung kann jedes Mitglied seine eigene Rangfolge an Präferenzen haben. Dasselbe gilt für jede Gruppe von Abgeordneten. Bei aufeinander folgenden Abstimmungen über mehrere Alternativen gibt es deswegen oft nicht eine, sondern wechselnde Mehrheiten, und sie können dann in einen endlosen Kreislauf münden. Ein Einzelner mag rationale, transitive Prioritäten haben und demgemäß den ersten Antrag gegenüber dem zweiten bevorzugen, den zweiten Antrag gegenüber dem dritten und deshalb den ersten Antrag auch gegenüber dem dritten. Ein Einzelner kann deshalb sinnvoll und logisch zwischen Alternativen wählen. Aber wenn die Prioritäten von einzelnen Abgeordneten in Gruppen zusammengezählt werden, ist diese Rationalität, das sinnvoll logische Entscheiden, nicht garantiert.

Angenommen ein Parlament, ein Ausschuss oder ein Arbeitskreis muss entscheiden, ob die Einkommens-, die Körperschafts- oder Umsatzsteuer erhöht wird. Bei der Abstimmung zwischen Paaren kann es leicht passieren, dass die Umsatzsteuer die Einkommenssteuer besiegt, die Körperschaftssteuer die Umsatzsteuer schlägt, aber die Einkommensteuer die Körperschaftssteuer. Aufgrund der ersten Paarung wissen wir, dass die Einkommenssteuer von der Umsatzsteuer besiegt werden kann, so dass der Kreislauf unendlich weitergehen könnte. Häufig gibt es nicht eine Mehrheit, sondern mehrere. Jede einzelne besteht aus verschiedenen Gruppen von Parlamentariern, von denen jeder einzelne seine eigene konsistente Prioritätenliste haben mag. Die Summe dieser Prioritäten kann aber inkonsistent und unstet sein, so dass zyklische Mehrheiten entstehen. 
Die Möglichkeit von Wahlzyklen ist seit langem bekannt. Ende des 18. Jahrhunderts wurde sie von dem französischen Philosophen, Politiker und Mathematiker Marquis de Condorcet beschrieben. Darauf fußt Kenneth Arrows mit einem Nobelpreis ausgezeichnete Theorie ${ }^{6}$, die aufzeigt, dass es keinen sicheren Weg gibt, die rationalen Präferenzen jedes Einzelnen in konsistente und stabile Präferenzen einer Gruppe zu übertragen - es sei denn, man verwendet nicht-mehrheitsgebundene Regeln für die Abstimmungen, zum Beispiel durch einen Wahlmodus, der die Reihenfolge der Abstimmung über Anträge bestimmt und die Zahl der Abstimmungen beschränkt.

Im britischen und in den meisten englischsprachigen Parlamenten, inklusive des USKongresses, wird ein Änderungsverfahren angewandt, in dem Alternativen miteinander verglichen werden. In jedem Wahlgang wird eine Alternative ausgeschlossen. In den meisten kontinentaleuropäischen Parlamenten wird über konkurrierende Anträge in Folge abgestimmt. Sobald eine Alternative eine Mehrheit erreicht hat, ist die Wahl beendet. Im Bundestag sind solche Abstimmungen über alternative Vorschläge relativ selten, denn in vielen Fällen gibt es nur eine Vorlage, die die Koalitionsfraktionen geschlossen unterstützen. Es hat aber schon bedeutende Ausnahmen gegeben. In diesen Fällen ist die Reihenfolge, in der abgestimmt wird, nicht immer dieselbe. Es ist allerdings ungeschriebene Regel, dass über die extremste Alternative als erstes abgestimmt wird und dann der Reihe nach über die Vorschläge, die immer weniger Neuerungen vorsehen. Auf jeden Fall hat der Modus, nach dem abgestimmt wird, starken Einfluss auf das Endergebnis. Erfahrene Partei- und Fraktionsführer wissen bestimmte Vorgänge zu ihrem eigenen Vorteil zu nutzen und versuchen, Entscheidungen mit einer „taktischen“ Abstimmungsstrategie zu beeinflussen. Sie halten bei jeder Abstimmung immer das Endergebnis im Blick, statt während jeder Etappe nach ihrer persönlichen Präferenz zu votieren.

Die Entscheidungen individueller Mitglieder können also nicht ohne komplizierte Regeln in Entscheidungen von Parlamenten übersetzt werden. Diese Regeln schaffen zwangsläufig Ungleichheit unter den Abgeordneten. Sie delegieren entscheidenden Einfluss an den Parlamentspräsidenten, an Fraktionsgeschäftsführer, an einen Ältestenrat oder an einen Geschäftsordnungsausschuss. Ohne solche Einrichtungen wären die Entscheidungen von Parlamenten inkonsistent, widersprüchlich und unstet.

Dennoch blicken die Bürger, die die Abstimmungen im Parlament beobachten, zynisch darauf. Naiverweise erwarten sie, dass es einfach sein sollte, Entscheidungen der einzelnen Abgeordneten in Entscheidungen des ganzen Parlamentes zu übersetzen. Aber das ist eben ganz und gar nicht einfach. So entsteht ein Konflikt zwischen dem Ideal der Mehrheit aus einzelnen Mitgliedern und den Realitäten der Entscheidungsfindung in großen Versammlungen. In Parlamenten wie dem Deutschen Bundestag, wo die meisten Abstimmungen entlang geschlossener Fraktionslinien verlaufen, verachtet die Öffentlichkeit ohnehin das, was sie als Fraktionszwang versteht.

\section{Das Paradox der Transparenz}

Repräsentation bedeutet im modernen demokratischen Staat die Wahl von Parlamentsmitgliedern durch die Bürger. In Ständegesellschaften konnten Repräsentanten sich selbst be-

6 Kenneth J. Arrow, Social Choice and Individual Values, New York 1951. 
rufen oder ihren Abgeordnetenstatus erben, so wie früher die Mitglieder des britischen Oberhauses. Heutzutage ist die Wahl in einem Wahlkreis zwingende Voraussetzung für Repräsentativität. Dieser Prozess wird selbst in Ländern nachgeahmt, die nicht für demokratisch erachtet werden. Repräsentation durch Wahl macht es notwendig, dass Wähler ihre Abgeordneten zur Verantwortung ziehen können. Also müssen sie in der Lage sein zu sehen, was Parlamentarier und Parlamente tun. Sitzungen müssen folglich offen und dem prüfenden Auge der Öffentlichkeit zugänglich sein. Demokratische Repräsentation impliziert Transparenz. Das deutsche Parlament hat dieses Prinzip bewusst in der Architektur des renovierten Reichstags symbolisiert.

Diese Transparenz gewährt der Öffentlichkeit aber Einblick in gerade jene Merkmale des Gesetzgebungsprozesses, die den Erwartungen der Bürger widersprechen. Transparenz erlaubt den Blick auf zeitraubende Verhandlungen zahlreicher Entscheidungsträger untereinander; auf Kompromisse über verschiedene Standpunkte, bei denen oft Prinzipien verloren zu gehen scheinen; auf unaufrechte Höflichkeiten der Abgeordneten untereinander in dem Versuch, Konflikte zu mildern; auf komplizierte Verfahren, um die Arbeit zu organisieren und Ergebnisse zu erzielen. Damit verkörpern Parlamente alles, was der demokratischen Öffentlichkeit an Politik missfällt: Die Zahl ihrer Mitglieder scheint übertrieben groß; sie arbeiten auf eine schwerfällige, anscheinend ineffiziente Art; es gibt Streit und Kompromisse; sie scheinen politische Initiativen zu blockieren und es nicht zu schaffen, die Probleme der Nation zu lösen; die Schwächen einzelner Abgeordneter verleiten manchmal zu der Annahme, alle seien korrupt.

Anders als in den Gerichten und der Regierung sind die Entscheidungsprozesse im Parlament relativ offen. Doch genau diese Transparenz ist der Ursprung des öffentlichen Misstrauens. John Hibbing und Elizabeth Theiss-Morse haben dieses Phänomen im amerikanischen Kontext untersucht und sind zu dem Schluss gekommen, dass „der Kongress von der Öffentlichkeit als ein Feind betrachtet wird (...), weil er so öffentlich ist"

Folglich versuchen Parlamente allerorts, Teile ihres Entscheidungsprozesses weniger zugänglich zu machen, indem viele Ausschüsse und die meisten internen Fraktionsgremien unter Ausschluss der Öffentlichkeit tagen. Natürlich verbergen sie auch oft die Verhandlungen zwischen der Exekutive und dem Parlament sowie zwischen Interessengruppen und Abgeordneten. Darum finden auch so wenige wirklich wichtige Angelegenheiten ihren Weg auf die Bühne des Parlaments, wo die Aufmerksamkeit der Öffentlichkeit sich konzentriert, und so verwundert es auch kaum, dass der Durchschnittsbürger verwirrt und besorgt ist.

Das Paradox der Transparenz treibt mitunter seltsame Blüten: Beispielsweise versuchen Abgeordnete, sich bei ihren Wählern beliebt zu machen durch Kritik an der Institution, der sie angehören. Abgeordnete und Fraktionen kritisieren das Parlament und legen ihren Kollegen alles zur Last, was die Öffentlichkeit am Parlament stört. Dies ermöglicht den Bürgern, gleichzeitig dem Parlament als Ganzes kritisch gegenüberzustehen und dennoch mit der Arbeit der ihnen nahe stehenden Fraktionen oder Abgeordneten zufrieden zu sein. Dies

7 John R. Hibbing / Elizabeth Theiss-Morse, Congress as Public Enemy: Public Attitudes Toward American Political Institutions, Cambridge 1995, S. 61. Für die Bundesrepublik vgl. Werner J. Patzelt, Warum verachten die Deutschen ihr Parlament und lieben ihr Verfassungsgericht? Ergebnisse einer vergleichenden demoskopischen Studie, in: ZParl, 36. Jg. (2005), H. 3, S. 517 $-538$. 
ist einer der Gründe, weshalb Mandatsträger in den Vereinigten Staaten so selten geschlagen werden, während die Unterstützung für den Kongress in der Öffentlichkeit in den letzten 35 Jahren nur zwischen 8 und 28 Prozent lag. ${ }^{8}$ Der Umgang mit der Transparenz bleibt immer eine Gratwanderung nach dem Motto: Wasch mir den Pelz, aber mach mich nicht nass.

\section{Das Paradox internationaler Vergleichbarkeit}

Als repräsentative Institutionen sind Parlamente in ihrer eigenen Kultur verwurzelt. Doch haben sie gemeinsame organisatorische Merkmale. Man kann sie vergleichen, messen und sogar als Modell anbieten.

Jenseits gewisser struktureller Gemeinsamkeiten unterscheiden sich Parlamente jedoch von Land zu Land. Abgeordnete werden durch verschiedene Wahlsysteme rekrutiert. Obwohl die Mitglieder der Parlamente nie schlichte stereotypische Abbilder ihrer Wählerschaft sind, haben sie viele landesspezifische Eigenschaften. Die Partei- beziehungsweise Fraktionsgruppierungen, die informellen Verhaltensregeln, ihre politische Macht - in all diesen Aspekten unterscheiden sie sich zwar, teilen aber die gleiche Herkunft, haben gemeinsame Vorfahren in den mittelalterlichen Parlamenten Europas.

In ihrer langen Geschichte haben Parlamente sich gegenseitig über politische und kulturelle Grenzen hinweg beeinflusst. Die Verbreitung bestimmter Verfahren und Gewohnheiten folgte im 17. und 18. Jahrhundert einem Weg von England nach Amerika. Im 19. Jahrhundert führte er vom britischen hin zu den französischen und belgischen Parlamenten, von dort aus nach Mitteleuropa und von den USA nach Lateinamerika. Im 20. Jahrhundert ging der institutionelle Transfer von den britischen und französischen Parlamenten in Richtung ihrer ehemaligen Kolonien sowie von west- und mitteleuropäischen Volksvertretungen hin zu den neuen Demokratien Osteuropas. Das Paradox besteht darin, dass Parlamente landesspezifisch sind, obwohl sie von Zeit zu Zeit voneinander Prozesse und Organisationsstrukturen kopiert haben oder aufgezwungen bekamen.

Es gab eine Zeit, da schien es, als würde Großbritannien Parlamente exportieren, dann waren es die USA. Im ausgehenden 20. Jahrhundert stand bemerkenswerterweise Deutschland Modell für viele Parlamentsarchitekten anderswo in Europa - auch wenn die Deutschen zurückhaltend waren, darauf stolz zu sein.

Wie sehr Parlamente sich zum „Exportartikel“ eignen, war schon immer eine Streitfrage. Sie erhielt besondere Aktualität durch die Verbreitung offener, konkurrenzfähiger politischer Systeme in Lateinamerika, Süd- und Osteuropa während der letzten 30 Jahre. Diese neuen Demokratien entwarfen ausnahmslos Parlamente als Teil ihres politischen Systems. Sie bauten auf ihren einheimischen, natürlich gewachsenen Versammlungsformen auf und ergänzten sie um einige Merkmale des US-Kongresses - im Falle der meisten lateinamerikanischen Länder - oder um bestimmte Merkmale westeuropäischer Parlamente - im Falle der Länder Süd- oder Ostmitteleuropas. Wir wissen wenig darüber, unter welchen Umständen diese neuen Körperteile vom Organismus angenommen oder abgestoßen werden, denn diese Exporte erzielten sehr unterschiedliche Ergebnisse. Während institutioneller Transfer

8 John R. Hibbing / Elizabeth Theiss-Morse, a.a.O., S. 38. 
und institutionelle Diffusion von West- nach Süd- und Osteuropa relativ gut gelang, kam es zu Misserfolgen in Afrika und dem Mittleren Osten. Schnittmuster lassen sich zwar exportieren; sollen sie im neuen Umfeld funktionieren, bedarf es der Anpassung an gewachsene politische Besonderheiten. Das birgt ungewisse Ergebnisse und unvorhergesehene Konsequenzen.

In den frühen Nachkriegsjahren gab es den Versuch eines institutionellen Transfers vom US-Kongress zum Bundestag. Eine Delegation des gerade neu gebildeten deutschen Parlaments war auf Studienreise 1949 in Washington zu Gast. Dort beeindruckte sie das Anhörungsverfahren des US-Kongresses. Ihre Begeisterung führte zur Übernahme der so genannten Hearings in die Regeln des Deutschen Bundestages im Jahre 1951. Während der folgenden 14 Jahre fanden nur an insgesamt neun Tagen Anhörungen statt (alle Ausschüsse eingerechnet). Im Jahre 1968 explodierte plötzlich ihre Zahl. Während der letzten Wahlperiode des Bundestags wurden an über 300 Tagen Hearings veranstaltet. Was war passiert? Aus Gründen, die in einem völlig anderen Zusammenhang standen, änderte sich in den 1960er Jahren das Verhältnis der Exekutive zur Legislative. Damit kam den Anhörungen eine politisch relevante Funktion zu, die sie zuvor nicht besessen hatten. Ohne das zugrunde liegende Muster des US-Kongresses hätte sich das Verfahren vielleicht gar nicht entwickelt; ohne die Veränderungen in Deutschlands politischer Landschaft wäre es nicht gediehen.

Die Geschichte institutioneller Verbreitung ist voller Beispiele des Transfers institutioneller Muster. Heute ist dieser Transfer nicht mehr wie einst auf Länder begrenzt, die geographisch beieinander liegen oder dieselbe Sprache sprechen. Die Geschwindigkeit, mit der Parlamente in den letzten 30 Jahren neu errichtet oder wieder errichtet wurden, ist in der Geschichte der Institution einzigartig. Politologen tun sich seit jeher schwer damit, die Determinanten für den Erfolg einer Parlamentstransplantation auszumachen. Das Paradox liegt darin, dass Parlamente einerseits die Politische Kultur ihres Landes widerspiegeln und darum so unterschiedlich sind wie ihre nationalen Kulturen. Andererseits haben sie alle genetische Merkmale, von denen sie sich nicht lösen können. Das Ergebnis kann eine $\mathrm{Mu}-$ tation sein. Manchmal ist diese interessant und vital, manchmal aber auch hinderlich. Das Scheitern der Parlamente Europas zwischen den Weltkriegen liefert viele Beispiele für ihre Sterblichkeit. Manche Parlamente haben sich selbst aufgelöst wie der Deutsche Reichstag im Jahre 1933. Andere wurden mit der Zeit so handlungsunfähig, dass sie zur leichten Beute von Diktatoren wurden.

\section{Skepsis gegenüber der Demokratie}

Parlamente sind mit offensichtlichen Widersprüchen konfrontiert, die aus ihrer Evolution von mittelalterlichen Ständevertretungen zu einem Instrument der Demokratie rühren. Diese Widersprüche lassen sich nicht lösen. Daraus ergeben sich einige besorgniserregende Konsequenzen. Einerseits haben Parlamente den Anspruch, demokratische, mehrheitsgebundene und egalitäre Institutionen zu sein. Andererseits müssen sie um ihrer Effektivität willen gegen diese Ansprüche verstoßen, sie mindestens relativieren: Sie müssen interne Hierarchien dulden; sie müssen Verfahren annehmen, die den Abstimmungsverlauf regulieren und das Mehrheitsprinzip einschränken; sie müssen ihren Entscheidungsprozess wenigstens teilweise vor ihren Wählern verbergen; sie müssen ihre jeweilige Nation repräsentieren und doch gleichzeitig bestimmte übernationale Strukturprinzipien befolgen. 
Diese Widersprüche geben Anlass für den Zynismus der Öffentlichkeit. Und da Parlamente ein Symbol der Demokratie sind und oft als Wegbereiter der Demokratisierung gedient haben, führen ihre inneren Widersprüche auch zu Skepsis gegenüber der demokratischen Regierungsform im Allgemeinen.

Misstrauen gegenüber der Politik ist in der demokratischen Welt weit verbreitet und mit der letzten Generation stetig gewachsen. Das hat zweifellos viele aktuelle Gründe: hartnäckige Probleme, denen sich die meisten Parlamente gegenübersehen, Skandale, die das Ansehen vieler prominenter Politiker zerstört haben, Mangel an eigener Regierungserfahrung in den neuen Demokratien. Politische Institutionen sollten kurzfristige Herausforderungen überstehen, Parlamente den Vertrauensschwund in die Regierung aushalten können. In vielen Ländern aber schwächen die inneren Widersprüche die Kapazität der Parlamente als Stützpfeiler der Demokratie.

Obwohl einzelne Abgeordnete oft bei ihren Wählern beliebt sind, führt das nicht zur Wertschätzung des Parlaments als Ganzes. Man ist sowohl in den USA als auch in Deutschland überzeugt, dass politische Bildung die Meinung der Öffentlichkeit zum Parlament verbessern kann. Jedoch gibt es keine stichhaltigen Beweise dafür, dass solche Anstrengungen auch die Beliebtheit der Institution steigern. Dies liegt nicht zuletzt daran, dass die hier aufgezeigten Missverständnisse der Bürger in den inhärenten Paradoxien der Institution selbst begründet sind. Der parlamentarische Entscheidungsprozess liegt einfach zu weit abseits der Erfahrung der meisten Menschen. Deswegen sollte man an eine Analogiebildung denken, um die Entscheidungsprozesse im Parlament mit denen in der Zivilgesellschaft und Alltagskultur zu verbinden.

Bundestagsführungen, Beschreibungen, wie ein Entwurf zum Gesetz wird, das menschliche Antlitz der Abgeordneten zeigen - all das führt meiner Meinung nach nicht weit genug. Wir sollten uns überlegen, wie wir den parlamentarischen Entscheidungsprozess mit den alltäglichen Erfahrungen der Menschen in Beziehung setzen können. In unseren Familien, bei der Arbeit, in Schulen und Kirchen - überall begegnen wir dem Problem, gemeinsam Entscheidungen zu treffen. In diesen Gruppen lernen wir, wie schwer es sein kann, sich von individuellen Präferenzen zu lösen und zu einem Entschluss der Gruppe zu gelangen. Dies sind die sozialen Dilemmata, die den Paradoxien der Parlamente zugrunde liegen. Wenn wir lernen, uns dieser Dilemmata bewusst zu werden, sie wiederzuerkennen, wenn sie uns zuhause begegnen, in Synagogen, Kirchen, Schulen und bei der Arbeit, dann können wir auf die Fähigkeiten zurückgreifen, die Robert Putnam unser „soziales Kapital“ genannt hat. Unsere Erfahrungen zuhause könnten uns helfen, den parlamentarischen Entscheidungsprozess zu verstehen und sogar zu schätzen. Wenn wir darauf aufmerksam machen, was Politologen das Dilemma des kollektiven Handelns nennen, erscheint der chaotische, unsaubere, ineffiziente und widersprüchliche Entscheidungsprozess im Parlament nicht mehr ganz so rätselhaft. Verständnis - und Respekt - für die parlamentarischen Paradoxien, die für das Funktionieren der Institution unaufhebbar sind, kann am besten erreicht werden, wenn wir Analogien bilden zwischen den Dingen, die wir um uns herum verstehen, und den Dingen, die uns auf politischer Ebene so erstaunen.

Die parlamentarische Regierungsform ist ohne Zweifel eine unwahrscheinliche Leistung unserer Zivilisation. Jene von uns, die den Parlamentarismus besonders schätzen, sind als Politiker, Wissenschaftler und Bürger verpflichtet, stets die parlamentarische Institution zu erklären und verständlich zu machen. 